\title{
Should scientists tell stories?
}

\author{
A narrative can effectively communicate scientific information. But when telling a perfect \\ story becomes an end in itself, the scientific process can be easily compromised.
}

Everyone loves a good story, and writers of many kinds use narrative techniques to get their message across. A recent Points of View article (Krzywinski and Cairo, Nat. Methods 10, 687, 2013) described how techniques of storytelling, such as a structured story arc, can effectively guide the presentation of scientific data in figures. But as pointed out in a Correspondence by Katz (p. 1045, this issue), the notion of communicating scientific information by storytelling can be taken too far.

The hypothetical scientist such as the one described by Katz, who allows a desired storyline to improperly influence experiments and who "embellishes" and "obfuscates" results, is clearly operating in a highly undesirable, even unethical, manner. But it is worth distinguishing between the use of rhetorical techniques as a tool for conveying information versus treating the telling of a scientific story as an end in itself.

Even so, are rhetorical techniques more likely to impede, rather than enable, a proper understanding of data? Should storytelling-setting the stage for the importance of an experiment, presenting the reader with an unknown or an unsolved problem, and interweaving interpretation with the reporting of results-have a role in communicating scientific results at all?

One may argue that in an idealized scholarly world, scientists should avoid storytelling. They should instead describe their original hypothesis, detail their experiments in the order conducted and present the data in the rawest form reasonable for interpretation. At the end they should state their conclusions.

But there are many problems with this scenario. Scientists are not automata and, in today's world, operate under substantial time pressures. Even if the scientist's colleagues in this idealized setting had the patience and time to navigate through a long, uninterpreted, purely factual exposition and to sufficiently grasp what was done and its significance, it would still be a cripplingly inefficient process. Furthermore, to borrow from the title of science historian Steven Shapin's recent book of essays, science is conducted by people "situated in time, space, culture and society, and struggling for credibility and authority." An argument for papers written purely as a factual blowby-blow account of experiments does not sufficiently take into account this reality.
Finally, a scientific paper is not a glorified laboratory notebook, that is, simply a record of what was done. Rather, it must place the research into a larger scientific context in addition to communicating the results and explaining its author's conclusions to other researchers so that they can assess and build on the findings.

Watson and Crick's Nature paper was a single page with one figure, no data and no methods-effectively a story, but a powerful one. Most research papers are far more extensive, but authors often shape the narrative to convey their arguments, presenting experiments in an order different from that in which they were conducted and leaving out aborted lines of inquiry and failed experiments. Some such liberties will be necessary to prevent readers from becoming hopelessly lost: research is a road on which one may take many wrong turns before a productive direction is found, and rarely must the reader also follow that process.

But there will be cases in which failed experiments bring a necessary nuance to the data, suggesting weaknesses in the argument or settings where the conclusions are questionable. Omission of such information may be unjustifiable. What is more, authors can easily segue into frank cherry-picking of data to support a desired conclusion, a practice that goes against the deepest goals of scientific research.

The rise of supplementary information has served an important function in providing a place for failed experiments and negative or unexplained results. Efforts by publishers to integrate supplementary information into the online version of the manuscript can crucially expose these data to readers without compromising a manuscript's narrative. But hard limits on supplementary information or efforts to eliminate it altogether could complicate a paper's narrative or, alternatively, whittle it down to a tightly told story with little room for more than one interpretation.

Determining how to handle data that do not fit a particular narrative and deciding how to describe one's results takes judgment; notably, this is judgment of a scientific, not a rhetorical, nature. When such a judgment is well made, exploiting the full range of one's abilities for exposition may illuminate, rather than hide, nuance in the results. After all, in science and out of it, the most interesting stories are often the complex, multilayered ones. 